\title{
LUMINOUS IR GALAXIES IN A MERGER SEQUENCE: BIMA CO IMAGING
}

\author{
Y. GAO AND R.A. GRUENDL \\ University of Illinois, Dept. of Astronomy \\ 1002 W. Green St., Urbana, IL 61801, USA \\ AND \\ C.-Y. HWANG AND K.Y. LO \\ Academia Sinica, IAA \\ P.O. Box 1-87, Nankang, Taipei, Taiwan 11529, ROC
}

\section{Introduction}

The power output in luminous infrared galaxies (LIGs, $L_{\mathrm{IR}} \gtrsim 10^{11} L_{\odot}, H_{0}=$ $75 \mathrm{~km} \mathrm{~s}^{-1} \mathrm{Mpc}^{-1}$ ) can approach the bolometric luminosity of quasars and can be provided by either starbursts or dust-enshouded QSOs, or both. Most LIGs appear to comprise of mergers of gas-rich galaxies. So, intense bursts of star formation apparently result from interaction and merging of galaxies, but the exact physical processes involved in collecting the large amount of gas involved and in initiating the starbursts are not well understood.

In order to trace observationally the conditions in the interstellar medium (ISM) that lead to starbursts, we have used the newly expanded Berkeley-Illinois-Maryland Association (BIMA) millimeter-wave array to map the molecular ISM in a sample of LIGs chosen to represent different phases of the interacting/merging process. Most importantly, a few LIGs in the sample might be in a "pre-starburst" phase, so they provide an ideal laboratory for studying the conditions leading to starbursts (Lo, Gao \& Gruendl 1997). Our emphasis is on the widely separated LIGs at early/intermediate stages of interaction for this reason, and also for complementary studies to previous CO imaging studies that have concentrated on relatively advanced merger systems (Scoville et al. 1991; Downes \& Solomon 1997 ) in which the ISM has already been highly disrupted by the interaction and starbursts. 
Our goal is to sample statistically the evolution of physical conditions of the molecular material in LIGs, and to delineate observationally the development of starburst activities along the merger sequence, using radio continuum, IR and other observations.

\section{Interacting Luminous Infrared Galaxies}

The observed decrease of $L_{\mathrm{CO}}$ with decreasing projected nuclear separation of a sample of interacting/merging LIGs can be interpreted as gas depletion resulting from the merger-enhanced starbursts (Gao \& Solomon 1998).

Unlike the morphologically selected "Toomre sequence", the LIGs in our sample have a comparable gas content so that the galaxies in the early stage are likely to have the gas reservoir to reach the ultraluminous starburst phase. Therefore, they are likely to be a better sample for the statistical study of interaction/merger induced starbursts leading to ultraluminous IR galaxies.

\section{CO Imaging of a Merger Sequence}

We present our $\mathrm{CO}$ images in Fig. 1 and the gas properties along a merger sequence in Table 1.

In Table 1, we list the sample galaxies roughly in a merger sequence including a few late stage mergers from literature (marked with ${ }^{*}$ ), thus a study of the molecular gas properties at various phases of the merging process can be performed. These are the much needed observations to test those sophisticated simulations (e.g., Barnes, this volume).

Clearly, a sequence of merging is observed in the molecular gas traced by $\mathrm{CO}$ :

(1). The morphology of the molecular gas changes from weakly disturbed and separate gas disks to the disturbed or merged-common-envelope gas disks and finally to a single common gas disk for the double nuclei of the two galaxies.

(2). The spatial $\mathrm{CO}$ extent drops from $\sim 20 \mathrm{kpc}$ for the early mergers to a few kpc for the intermediate mergers. Advanced mergers have typical nuclear CO concentration $\lesssim 1 \mathrm{kpc}$.

(3). The corrected face-on central gas surface density increases from a few times $10^{2} M_{\odot} \mathrm{pc}^{-2}$ to $>10^{3} M_{\odot} \mathrm{pc}^{-2}$ in our sample. However, advanced ultraluminous mergers typically have $>10^{4} M_{\odot} \mathrm{pc}^{-2}$. A rapid increase of the nuclear gas surface density is evident along the sequence.

(4). The $L_{\mathrm{IR}} / M\left(\mathrm{H}_{2}\right)$ ratio (a measure of star formation efficiency, SFE) increases by roughly several times from the early mergers to the intermediate/advanced mergers. We can estimate the central SFE ratio, by scaling the far-IR luminosity and extent with those of the radio continuum emis- 
TABLE 1. Luminous Infrared Galaxies in a Merger Sequence.

\begin{tabular}{lcrcccccc}
\hline Source & $\begin{array}{c}R_{\mathrm{Sep}}^{1} \\
\mathrm{kpc}\end{array}$ & $\begin{array}{r}L_{\mathrm{IR}} \\
10^{11} L_{\odot}\end{array}$ & $\begin{array}{c}M\left(\mathrm{H}_{2}\right)^{2} \\
10^{10} M_{\odot}\end{array}$ & $\begin{array}{c}\text { Beam } \\
\prime \text { kpc }\end{array}$ & $\mathrm{CO}^{3}$ & $\begin{array}{c}\Sigma_{\mathrm{H}_{2}} \\
10^{3} M_{\odot} \mathrm{pc}^{-2}\end{array}$ & $\begin{array}{c}\left.L_{\mathrm{IR}} / M_{(} \mathrm{H}_{2}\right) \\
L_{\odot} / M_{\odot}\end{array}$ \\
\hline Arp302 & 25.8 & 4.1 & 8.0 & 6.0 & 3.7 & $\mathrm{u}+\mathrm{e}$ & 0.4 & 5.0 \\
N6670 & 14.6 & 3.8 & 5.5 & 4.8 & 2.7 & $\mathrm{u}+\mathrm{e}$ & 0.3 & 6.9 \\
U2369 & 13.1 & 3.9 & 3.4 & 6.1 & 3.6 & $\mathrm{u}+\mathrm{e}$ & 1.2 & 11.5 \\
Arp55 & 10.7 & 4.7 & 5.8 & 4.4 & 3.1 & $\mathrm{u}+\mathrm{e}$ & 1.3 & 8.1 \\
VV114* & 6.0 & 4.2 & 5.1 & 3.7 & 1.4 & $\mathrm{e}$ & 3.3 & 8.2 \\
N5256 & 4.8 & 3.1 & 2.7 & 4.6 & 2.3 & $\mathrm{u}+\mathrm{e}$ & $>1.3$ & 11.5 \\
Mrk848 & 4.7 & 7.2 & 3.4 & 3.9 & 2.9 & $\mathrm{u}+\mathrm{e}$ & $>1.0$ & 21.2 \\
Arp299* & 4.5 & 6.4 & 1.4 & 2.3 & 0.4 & $\mathrm{e}$ & 11.3 & 45.0 \\
N6090 & 3.5 & 3.0 & 2.4 & 1.9 & 1.0 & $\mathrm{e}$ & 3.0 & 12.5 \\
N1614 & 2.0 & 4.1 & 1.6 & 5.8 & 1.7 & $\mathrm{u}$ & $>1.5$ & 25.6 \\
Mrk273* & 1.0 & 13.2 & 2.7 & 2.3 & 1.5 & $\mathrm{u}+\mathrm{e}$ & 5.5 & 47.6 \\
Arp220* & 0.4 & 15.0 & 3.0 & 1.0 & 0.4 & $\mathrm{e}$ & 80 & 50.0 \\
Mrk231* & 0.0 & 30.4 & 3.5 & 0.9 & 0.7 & $\mathrm{u}+\mathrm{e}$ & 30 & 77.2 \\
\hline
\end{tabular}

${ }^{1}$ Projected separation between the two galaxy nuclei. ${ }^{2} 4.78 L_{\mathrm{CO}} \mathrm{K} \mathrm{km} \mathrm{s}^{-1} \mathrm{pc}^{2}$, the single-dish CO luminosity. ${ }^{3} \mathrm{CO}$ morphology, $\mathrm{u} \equiv$ unresolved peak; $\mathrm{e} \equiv$ extended structures resolved by the beam.

sion or the mid-IR emission (Hwang et al. 1998), which tends to increase more drastically than the global SFE along the sequence.

(5). We found that early stage mergers or pre-mergers like Arp 302 and NGC 6670 appear to have much smaller SFE throughout the entire interacting/merging disks, comparable to that of GMCs in the Milky Way disk. This strongly suggests that LIGs in the pre-merging stage are in a pre-starburst phase (Lo, Gao \& Gruendl 1997).

(6). The starburst phase as indicated by large SFE's seems to start once the molecular gas disks begin merging and the ultraluminous starburst phase seems to be occurring only after the molecular gas disks merge into a common disk in late stage mergers.

(7). Gas seems to respond faster than the stellar component when merging advances to late stage (i.e., the gas disks merge faster than the stellar disks).

\section{References}

Downes, D., \& Solomon, P.M. 1997, ApJ, submitted

Gao, Y., \& Solomom, P.M. 1998, ApJ, submitted

Hwang, C.Y. et al. 1998, this volume

Lo, K.Y., Gao, Y., \& Gruendl, R.A. 1997, ApJ, 475, L103

Scoville, N.Z. et al. 1991, ApJ, 370, 158 

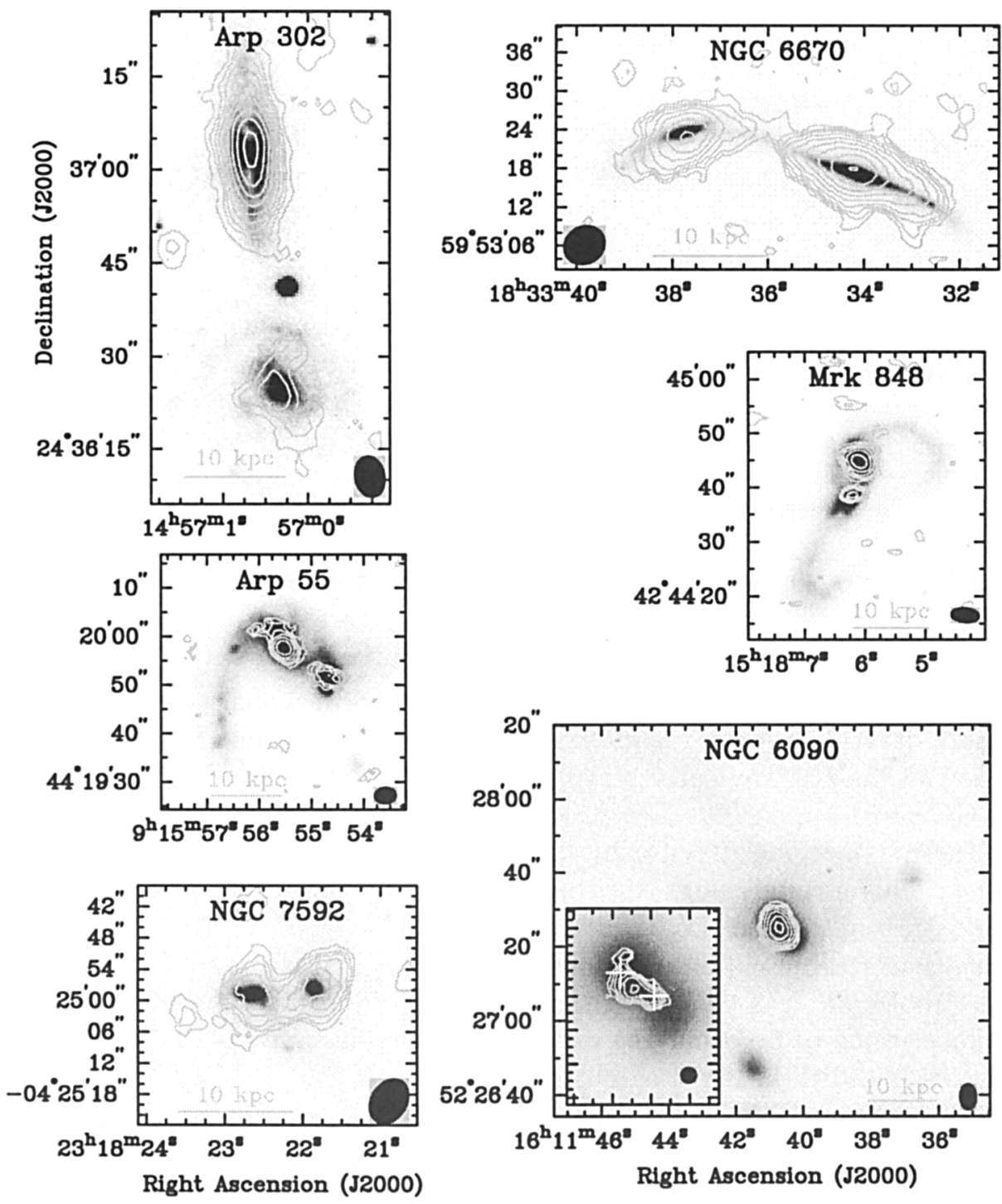

Figure 1. A merger sequence leading to ultraluminous starburst (CO contours overlaid on optical images). The insert in N6090 shows higher resolution $\left(\sim 2^{\prime \prime}\right)$ CO contours with two radio continuum peaks marked. 